\title{
Exciton-Phonon Coupling in the Ultraviolet Absorption and Emission Spectra of Bulk Hexagonal Boron Nitride
}

\author{
Fulvio Paleari, ${ }^{1, *}$ Henrique P. C. Miranda, ${ }^{1,2}$ Alejandro Molina-Sánchez, ${ }^{3}$ and Ludger Wirtz ${ }^{1}$ \\ ${ }^{1}$ Physics and Materials Science Research Unit, University of Luxembourg, \\ 162 a avenue de la Faïencerie, L-1511 Luxembourg, Luxembourg \\ ${ }^{2}$ Institute of Condensed Matter and Nanosciences, Université catholique de Louvain, \\ Chemin des étoiles 8, bte L7.03.01, 1348, Louvain-la-Neuve, Belgium \\ ${ }^{3}$ Institute of Materials Science (ICMUV), University of Valencia, Catedrático Beltrán 2, E-46980 Valencia, Spain
}

(Received 15 October 2018; revised manuscript received 5 February 2019; published 7 May 2019)

\begin{abstract}
We present an ab initio method to calculate phonon-assisted absorption and emission spectra in the presence of strong excitonic effects. We apply the method to bulk hexagonal BN, which has an indirect band gap and is known for its strong luminescence in the UV range. We first analyze the excitons at the wave vector $\bar{q}$ of the indirect gap. The coupling of these excitons with the various phonon modes at $\bar{q}$ is expressed in terms of a product of the mean square displacement of the atoms and the second derivative of the optical response function with respect to atomic displacement along the phonon eigenvectors. The derivatives are calculated numerically with a finite difference scheme in a supercell commensurate with $\bar{q}$. We use detailed balance arguments to obtain the intensity ratio between emission and absorption processes. Our results explain recent luminescence experiments and reveal the exciton-phonon coupling channels responsible for the emission lines.
\end{abstract}

DOI: 10.1103/PhysRevLett.122.187401

Hexagonal boron nitride (hBN) is well known for its strong luminescence signal in the UV range [1-3]. The fine structure of both emission and absorption spectra has been under heavy discussion until very recently. First-principles calculations agree upon the existence of an indirect quasiparticle band gap from the area around the $K$ point to the $M$ point of the hexagonal Brillouin zone (BZ) [4], with the direct band gap lying $0.5 \mathrm{eV}$ higher [Fig. 1(a)]. However, the layered structure with a quasi-2D confinement of electron-hole pairs within the layer and reduced screening outside leads to the formation of strongly bound excitons $[4,5]$. In experimental absorption spectra [6], a strong peak is seen around $6 \mathrm{eV}$, which is interpreted by first-principles calculations using many-body perturbation theory as an exciton with a huge binding energy of $700 \mathrm{meV}$. This direct exciton lies below the bottom of the conduction band [black dashed line in Fig. 1(a)]. Therefore, the optical gap of hBN has often been considered to be "direct," despite experimental observation of a fine structure appearing in both absorption [1] and emission spectra [7,8]. Symmetry-breaking effects such as JahnTeller distortion in the excited state [8] or the interaction of excitons with point defects [9] were invoked to explain the fine structure.

Recently, with the help of photoluminescence on highpurity samples, Cassabois, Valvin, and Gil [10] explained the fine structure in terms of recombination of electrons from the conduction band minimum to the valence band maximum, assisted by emission of phonons with the (a)

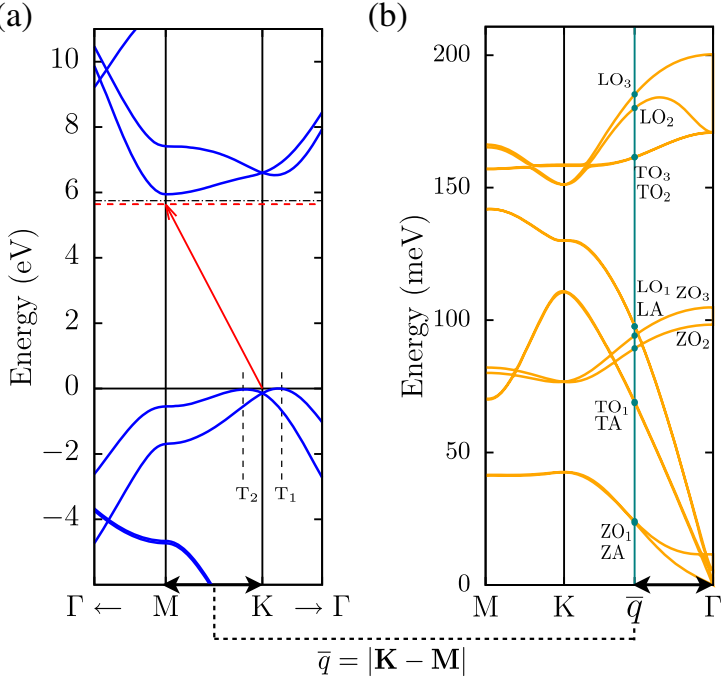

FIG. 1. Finite- $q$ calculations in hexagonal boron nitride. (a) $G W_{0}$ quasiparticle band structure of bulk hBN, displayed in the relevant part of the BZ. The dashed horizontal black line represents the position of the lowest-bound direct exciton, while the dashed red lines correspond to the indirect excitons of momentum $\bar{q}=|K-M|$. The exact valence band maxima are labeled $T_{1}$ and $T_{2}$. (b) The phonon dispersion in bulk hBN. The teal vertical line highlights the frequencies of the phonon modes with momentum $\bar{q}$. The notation is $Z$ for out-ofplane modes, $L$ for in-plane longitudinal modes, and $T$ for in-plane transverse modes. $O(A)$ stands for optical (acoustic) modes. 
corresponding wave vector $\bar{q}$ (see Fig. 1). Several new publications followed up on this topic, cementing this interpretation [11-14] notably through the mass dependence of the position of phonon-assisted emission peaks in isotopically clean samples [15]. Yet, the proposed interpretation is on the level of independent electrons and holes, insufficient to account for the strong Coulomb interaction in $\mathrm{hBN}$ and thus neglecting excitonic effects. Clearly, the position of the direct exciton is below the conduction band minimum in Fig. 1. However, excitonic states with finite wave vector $\bar{q}$ can have an energy below the direct exciton (horizontal red dashed line). The position of these indirect excitons and how they contribute to optical absorption and luminescence via emission and absorption of phonons of wave vector $\bar{q}$ are the topic of this Letter. For this purpose, we have developed a theory and computational recipe which explains quantitatively the optical spectra of hBN and which can also be used to compute the optical spectra of other materials with an indirect (optical) gap.

$A b$ initio calculations of indirect absorption are still sparse and, so far, restricted to the independent-particle picture. Notably, indirect absorption was calculated for silicon [16-19], where the independent-particle picture is a good approximation. A unified approach to describe on the same footing lattice-dependent band features (such as band gap renormalization with temperature) and phonon-assisted transitions was proposed [17-19]. However, it is computationally expensive and restricted to static approximations. Moreover, various models of the exciton-phonon coupling including dynamical effects are available in the literature [20-26] (with Ref. [27] specifically tackling luminescence), although their direct test on real materials from first principles is, again, computationally demanding. Here, we present a computational scheme that captures the exciton-phonon coupling in a finite-difference approach and treats luminescence intensities using detailed balance arguments. We explain the experimental PL spectra in terms of phonon-assisted recombination from finitemomentum excitonic states.

Hexagonal $\mathrm{BN}$ is a layered material with a honeycomb lattice in $A A^{\prime}$ stacking, possessing $D_{6 h}$ space group symmetry. Using experimental lattice parameters [28], we first compute the direct excitons using the primitive unit cell. Starting with the independent-particle band structure at the density-functional theory (DFT) level, we obtain the quasiparticle band structure through a semi-selfconsistent $G W_{0}$ scheme, converging iteratively only the eigenvalues entering the Green's function. This procedure is used to correct the usual quasiparticle gap underestimation given by the $G_{0} W_{0}$ approximation [29] in BN systems [30]. We obtain a direct quasiparticle gap of $6.46 \mathrm{eV}$ and an indirect one of $5.96 \mathrm{eV}$ [Fig. 1(a)]. In the following, we will approximate the momentum difference of the indirect band gap as $\bar{q}=|\mathbf{K}-\mathbf{M}|$. A discussion on this approximation with respect to the exact positions of the valence band maxima [31] [Fig. 1(a)] can be found in Supplemental Material [32], Sec. III.

Solving the Bethe-Salpeter equation (BSE) [44], we obtain two doubly degenerate excitonic states at $q=0$ which form a Davydov pair $[45,46]$ due to interlayer interaction [30]: The first exciton at $5.70 \mathrm{eV}$ has $E_{2 g}$ symmetry and is thus dark. The second exciton at $5.75 \mathrm{eV}$ (gray vertical line in Fig. 2) possesses $E_{1 u}$ symmetry (odd under inversion) and is thus bright. (More on the group theory can be found in Sec. VI of Supplemental Material [32] and Ref. [30]. Note that we are dealing only with light polarized parallel to the $\mathrm{BN}$ planes which dominates the optical spectra.)

In order to study finite momentum $\bar{q}$ excitons, we perform the same calculations on a special hBN supercell, containing 12 atoms per layer and chosen such that $\bar{q}$ will be folded onto $\Gamma$ in its new Brillouin zone [47] (see Supplemental Material [32]). Two additional excitonic states, $i 1$ and $i 2$ (dashed red lines in Fig. 2) at 5.63 and $5.65 \mathrm{eV}$, respectively, appear below the lowest-bound direct exciton (in agreement with recent results $[14,48]$ and confirming that not only the quasiparticle gap but also the optical gap of bulk hBN is "indirect"). These states originate from the splitting, at finite momentum, of the doubly degenerate $E_{2 g}$ exciton. The $i 1$ exciton transforms as the $B_{1}$ representation of the $C_{2 v}$ symmetry group, and $i 2$ transforms as $A_{1}$ (see Supplemental Material [32]). These finite- $q$ states are dark by themselves but candidates for phonon-assisted absorption and emission. The same symmetry considerations can be found in the phonon dispersion [Fig. 1(b)]: At $\Gamma$, the in-plane phonon modes form Davydov pairs and transform according to the $E_{1 u}$ and $E_{2 g}$

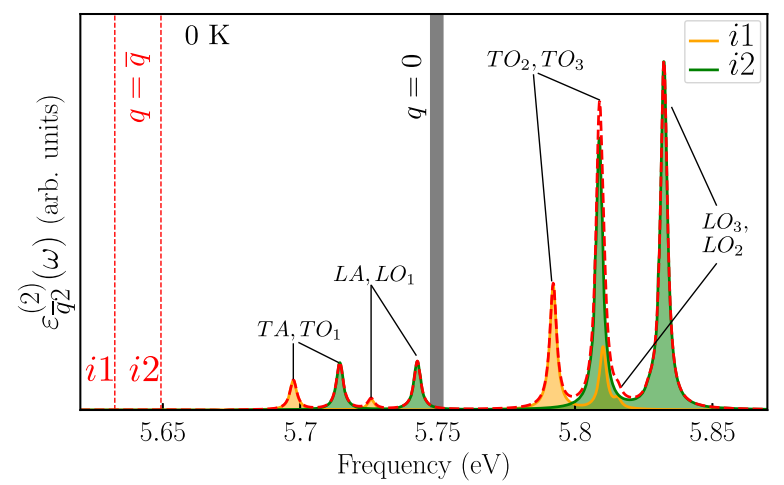

FIG. 2. Phonon-assisted absorption in hexagonal boron nitride. Imaginary part of the dielectric function including contributions of transitions at $q=\bar{q}$ mediated by a single phonon [dashed red line, $\varepsilon_{\bar{q} 2}^{(2)}(\omega)$ in the text]. The orange (green) peaks originate from the phonon couplings to $i 1$ ( $i 2)$, and the phonon modes responsible for them are labeled. The peak broadenings are set to $1.5 \mathrm{meV}$ for the temperature of $0 \mathrm{~K}$. The dashed red vertical lines indicate the positions of the two pairs of $A_{1}+B_{1}$ excitons at $\bar{q}$ (labeled $i 1$ and $i 2$ ). The thick gray vertical line is at the position of the main optically active $q=0$ exciton. 
representation, respectively. Along $\Gamma K$, these representations reduce to $A_{1}$ and $B_{1}$. According to group theory (see Supplemental Material [32]), the $B_{1}$ exciton can couple to $B_{1}$ phonons and the $A_{1}$ exciton can couple to $A_{1}$ phonons if the light polarization $\mathbf{e}$ is parallel to $\bar{q}$. For light polarization perpendicular to $\bar{q}$, the $B_{1}$ exciton couples to $A_{1}$ phonons and the $A_{1}$ exciton to $B_{1}$ phonons. Coupling with out-ofplane modes is forbidden [49].

Having understood the symmetry constraints for coupling between finite- $q$ excitons and phonons, we now derive a general expression to calculate these couplings and, thus, phonon-assisted optical spectra. We address this task by (i) using a static approximation for the exciton formation probability and (ii) restricting the coupling to harmonic phonons with momentum $\bar{q}$. In our calculations, we focus on the energy region close to $i 1$ and $i 2$. We proceed by considering the complex dielectric function $\varepsilon(\omega)=\varepsilon_{1}(\omega)+i \varepsilon_{2}(\omega)$, which describes the linear response of the system and is closely related to the experimentally measurable absorption coefficient $\alpha(\omega)=$ $\omega \varepsilon_{2}(\omega) / c n_{r}[\varepsilon(\omega)]\left(n_{r}\right.$ being the refractive index). A calculation with the atoms clamped at the equilibrium positions yields $\varepsilon_{2}^{(0)}(\omega)=C \sum_{S} \mid T^{S}{ }^{2} \operatorname{Im}\left\{1 /\left(\hbar \omega-E^{S}+i \eta\right)\right\}$, where $\left|T^{S}\right|^{2}$ is the formation probability of exciton $S$ and $C$ is a dimensional constant (Sec. I in Supplemental Material [32]). The denominator determines the peak structure of $\varepsilon_{2}$, with $E^{S}$ being the exciton energy and $\eta$ the exciton linewidth. In order to obtain the response due to the indirect excitons, we consider the Taylor expansion of $\varepsilon(\omega)$ up to second order in the atomic displacements [19]. This gives a static correction to the equilibrium response $\varepsilon(\omega)=\varepsilon^{(0)}(\omega)+\varepsilon_{\bar{q}}^{\text {st, }(2)}(\omega)$, in which the term (Sec. V in Supplemental Material [32])

$$
\varepsilon_{\bar{q}}^{\mathrm{st},(2)}(\omega)=\frac{1}{2} \sum_{\lambda}\left(\left.\sum_{i}^{N_{\bar{q}}} \frac{1}{2} \sum_{j}^{2} \frac{\partial^{2} \varepsilon_{j}^{(0)}(\omega)}{\partial R_{\lambda \bar{q}_{i}}^{2}}\right|_{\mathrm{eq}}\right) \sigma_{\lambda \bar{q}}^{2}
$$

adds the contribution of transitions assisted by a single phonon of momentum $\bar{q}$. In this expression, $j$ represents the polarization direction of the incoming light, over which we average, and $i$ labels each of the $N_{\bar{q}}=6$ equivalent $\bar{q}$ vectors in the $\mathrm{BZ}$, over which we sum. $R_{\lambda \bar{q}_{i}}$ refers to a set of atomic displacements according to phonon mode $\lambda$ with momentum $\bar{q}_{i}$ (indicated with a vertical teal line in the phonon dispersion plot in Fig. 1). The quantity $\partial^{2} \varepsilon_{j}^{(0)}(\omega) /$ $\partial R_{\lambda \bar{q}_{i}}^{2}$ is evaluated by finite displacement from the equilibrium atomic positions. The last factor is the thermal average of the squared displacement of a quantum harmonic oscillator, given by $\sigma_{\lambda \bar{q}}^{2}=l_{\lambda \bar{q}}^{2}\left[2 n_{B}\left(\Omega_{\lambda \bar{q}}, T\right)+1\right]$, with $n_{B}$ being the Bose-Einstein distribution for phonons and $l_{\lambda \bar{q}}^{2}=\hbar /\left(2 M_{\lambda \bar{q}} \Omega_{\lambda \bar{q}}\right)$ [50]. Even though the function $\varepsilon_{\bar{q}}^{\mathrm{st},(2)}(\omega)$ includes contributions from the derivatives of both $T^{S}$ and $E^{S}$, for indirect transitions only one term survives in its imaginary part:

$\left.\operatorname{Im} \frac{\partial^{2} \varepsilon^{(0)}(\omega)}{\partial R_{\lambda \bar{q}}^{2}}\right|_{\mathrm{eq}}=\left.C \sum_{S^{\prime}} \frac{\partial^{2}\left|T^{S^{\prime}}\right|^{2}}{\partial R_{\lambda \bar{q}}^{2}}\right|_{\mathrm{eq}} \operatorname{Im}\left\{\frac{1}{\hbar \omega-E^{S^{\prime}}+i \eta}\right\}$,

where $S^{\prime}$ restricts the sum to the $q=\bar{q}$ excitons. This result allows us to reintroduce the phonon frequency dependence of $\varepsilon_{\bar{q}}^{(2)}(\omega)$ by imposing the correct energy conservation from the perturbation theory and distinguishing between phonon emission $\left(\propto n_{B}+1\right)$ and phonon absorption $\left(\propto n_{B}\right):\left[2 n_{B}+\right.$ $1] /\left(\hbar \omega-E^{S^{\prime}}+i \eta\right) \rightarrow\left[n_{B}+1\right] /\left(\hbar \omega-E^{S^{\prime}}-\hbar \Omega_{\lambda \bar{q}}+i \eta\right)+$ $n_{B} /\left(\hbar \omega-E^{S^{\prime}}+\hbar \Omega_{\lambda \bar{q}}+i \eta\right)$. Renaming the numerator between large parentheses in Eq. (1) (including the 1/2 factors) as $\left|t_{\lambda \bar{q} S^{\prime}}^{\text {static }}\right|^{2}$, since it represents the static formation probability of exciton $S^{\prime}$ mediated by a phonon mode $\lambda$ with momentum $\bar{q}$ and frequency $\Omega_{\lambda \bar{q}}$, we obtain the final expression:

$\varepsilon_{\bar{q} 2}^{(2)}(\omega)=\sum_{\lambda S^{\prime}}\left|t_{\lambda \bar{q} S^{\prime}}^{\text {static }}\right|^{2} l_{\lambda \bar{q}}^{2}\left[n_{B}+1 / 2 \mp 1 / 2\right] \delta\left(\hbar \omega-E^{S^{\prime}} \pm \hbar \Omega_{\lambda \bar{q}}\right)$.

Here, the upper (lower) sign refers to the process of phonon absorption (emission).

Applying Eq. (3) to the description of the process of exciton formation via photon absorption together with phonon emission at $T=0 \mathrm{~K}$ and computing the derivatives of $\varepsilon(\omega)$ with finite-difference DFT-BSE calculations in the supercell, we obtain the spectrum in Fig. 2. It is possible to identify a multipeak structure associated to the coupling of both the $i 1$ and $i 2$ excitons to all the in-plane phonon modes, with the higher-energy state $i 2$ accounting for most of the oscillator strength. The coupling of $i 1$ and $i 2$ with specific phonon modes depends on the light polarization direction according to the symmetry selection rules detailed in Sec. VI of Supplemental Material [32]. Since the phonon frequencies are close to the energy difference between direct and indirect excitons, the phonon-assisted peaks are distributed around the brightest direct exciton peak with most of the oscillator strength remaining in this narrow energy range. This result suggests that phonon-assisted absorption in $\mathrm{hBN}$ is at the origin of the fine structure observed around the brightest exciton peak in absorption experiments $[1,14]$. Notice, however, that in experiments, the indirect contribution to the optical absorption spectrum will not appear as a series of discrete peaks (as in our approximation) but rather be a continuous function resulting from the integration over all $q$ vectors in the $\mathrm{BZ}$ (since they can be connected to the $q=0$ states by phonons of different momentum).

We now turn to the calculation of luminescence spectra. Here, we will neglect all out-of-equilibrium processes 
[51-53] and assume all excited carriers relaxed to the bottom of the excitonic dispersion curves. We take the probabilities of exciton formation and annihilation to be the same (i.e., detailed balance of phonon-assisted optical processes), a reasonable assumption in our scheme as we are computing the transition rates in a static approximation. This enables us to employ the van Roosbroeck-Shockley (RS) relation to compute the spontaneous emission rate $R^{\mathrm{sp}}(\omega)$ starting from the absorption coefficient [54], which entails two common experimental assumptions: (i) The system is in a steady state, with the contribution from stimulated emission being very small; (ii) in the steady state, the occupation functions of the excitonic states involved in recombination processes can be approximated with those at thermal equilibrium. The energy of an emitted photon differs from the one of an absorbed photon (indirect absorption with phonon emission) by twice the frequency of the phonon involved. We thus define $\varepsilon_{\lambda \bar{q}}^{\mathrm{em}}(\omega)=\varepsilon_{\lambda \bar{q}}^{(2)}\left(\omega-2 \Omega_{\lambda \bar{q}}\right)$. Because of this, each phonon-assisted peak is mirrored with respect to the energy of the excitonic state involved. To apply the RS relation, originally derived in the context of direct independentparticle transitions [55], we have to extend it to excitons (see Sec. VII of Supplemental Material [32]): In the lowdensity limit, this can be approximated by taking the occupation function for excitons as the Boltzmann factor $N_{B}\left(\Delta E_{i 1, i}\right)=e^{-\left(E^{i}-E^{i 1}\right) / k_{B} T_{\text {exc }}}$ ( $E^{i 1}$ being the energy minimum of the exciton dispersion curve and $E_{i}$ the energy of any exciton $i$ ):

$R_{\bar{q}}^{\mathrm{sp}}(\omega)=\sum_{\lambda} \frac{\omega\left(\omega+2 \Omega_{\lambda \bar{q}}\right)^{2}}{\pi^{2} \hbar c^{3}} n_{r}(\omega) \operatorname{Im}\left\{\varepsilon_{\lambda \bar{q}}^{\mathrm{em}}(\omega)\right\} N_{B}$.

It is sufficient to compute the refractive index from $\varepsilon^{0}(\omega)$, which completely determines its slow decay at low frequencies. Because of the large energy difference between $E^{i 1}$ and the main direct peak, the latter will always be suppressed by the Boltzmann factor $N_{B}$ up to room temperature, and therefore it should not be seen in a PL experiment. The results for $R_{\bar{q}}^{\mathrm{sp}}(\omega)$ are plotted in Fig. 3(a). The energy differences between excitonic levels $\left(\Delta E_{i 1, i 2}=\right.$ $17 \mathrm{meV}$ ) and the phonon frequencies (from 50 to $200 \mathrm{meV}$ ) are large, giving rise to a well-spaced peak structure that can be easily resolved. We notice two separated groups of features which are clearly seen in the experiment [Fig. 3(b), black dots]: one at higher energy generated by lowfrequency phonon emission, and the other at lower energy due to high-frequency optical phonons. The emission spectrum is almost completely dominated by the lowestbound exciton $i 1$, since the occupation factor quenches most of the peaks related to $i 2$. However, experiments have shown [10] that in bulk hBN the excitonic temperature $T_{\text {exc }}$ that goes into the Boltzmann factor $N_{B}$ is greater than the lattice temperature $T_{L}$. If we set $T_{\mathrm{exc}}=T_{L}=10 \mathrm{~K}$ in $R_{\bar{q}}^{\mathrm{sp}}$,
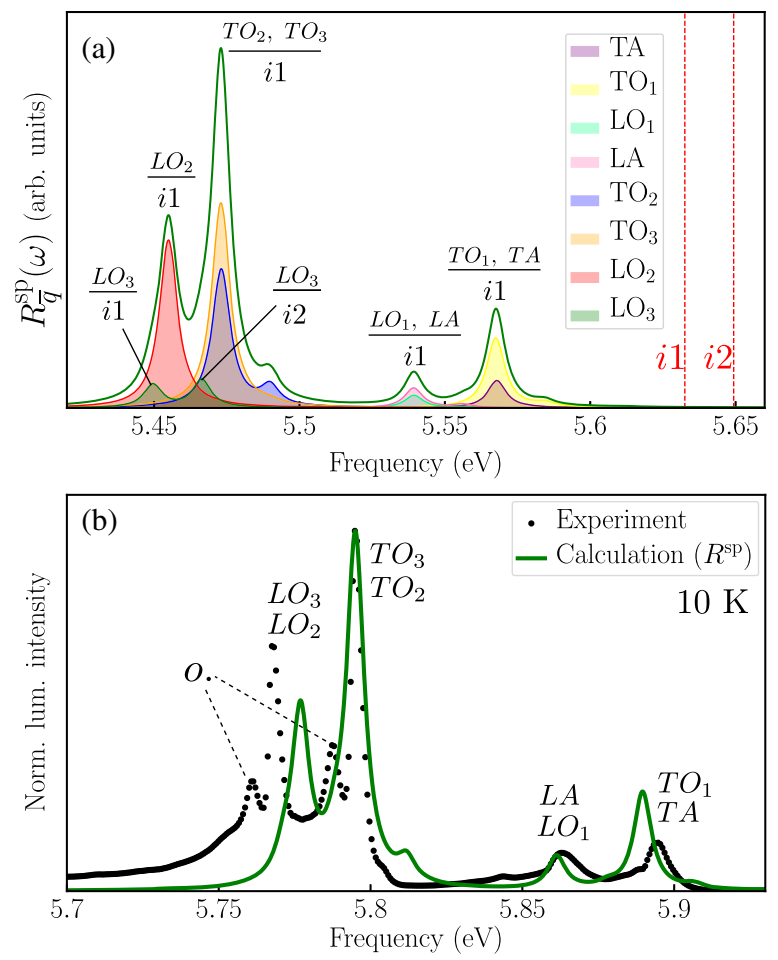

FIG. 3. Phonon-assisted emission in bulk hexagonal boron nitride. (a) Spectral function $R_{\bar{q}}^{\mathrm{sp}}=\sum_{\lambda} R_{\lambda \bar{q}}^{\mathrm{sp}}$ (green solid line) at $5 \mathrm{~K}$. The $\lambda$ components of the spectrum, belonging to the different phonon modes, are also plotted in various colors. The excitonphonon couplings are labeled. (b) Comparison with the normalized experimental spectrum (black dots) at $10 \mathrm{~K}$ from Ref. [14]. The multiphonon overtones are denoted as "o." Notice that here our spectrum is blueshifted by $0.322 \mathrm{eV}$ to match the experimental one. The temperature-dependent peak widths are described according to a linear model [23] (parameters taken from the experimental fit; see Supplemental Material [32]). The experimental excitonic temperature $T_{\mathrm{exc}}=55 \mathrm{~K}$ (see the text) is used.

we obtain only the peaks coming from $i 1$, while setting $T_{\text {exc }}$ to the experimental value of $55 \mathrm{~K}$ [as in Fig. 3(a)] leads to the appearance of the quenched peaks from $i 2$. On the experimental side, the peaks due to high-frequency modes are clearly visible in Fig. 3(b) at $10 \mathrm{~K}$ : The separation between these peaks thus corresponds to the separation between the LO and TO modes at point $\bar{q}$ in the theoretical calculations, while in the experiment it corresponds to the splitting at point $\bar{q}+\Delta q$, where $\Delta q$ is the error committed by approximating the "true" $q$ point $\left|T_{1}-M\right|$ with $\bar{q}=|K-M|$. We attribute to this discrepancy the difference of $\sim 8 \mathrm{meV}$ between the theoretical and experimental peak separations [56]. In our results, the quasidegenerate transverse modes $\mathrm{TO}_{2}$ and $\mathrm{TO}_{3}$ couple with similar strength to $i 1$, whereas the longitudinal mode $\mathrm{LO}_{3}$ has a higher frequency than $\mathrm{LO}_{2}$ and a much weaker coupling to $i 1$ (in the absorption case, the coupling of mode $\mathrm{LO}_{3}$ with $i 2$ is the strongest one). As expected, we cannot capture the various satellite peaks in Fig. 3(b), which experimentally 
are assigned to multiphonon processes involving zonecenter shear phonon modes [57].

In conclusion, we have presented a theoretical and computational approach to phonon-assisted absorption and emission in bulk $\mathrm{hBN}$ where very strong excitonic effects are present. Using a supercell for finite- $q$ excitons and a finite-difference method for exciton-phonon coupling, we are able to reproduce the single-phonon-assisted spectral features obtained in luminescence experiments. The structure of the emission spectrum can be understood in terms of a Davydov pair of finite- $q$ excitons coupling with different strengths to the various in-plane phonon modes. The method can be applied, in principle, to any material with an indirect gap. It is particularly suited for materials with strong excitonic effects as they often occur in layered materials. For example, it could be applied to bilayers of $\mathrm{MoS}_{2}$ or to the case of monolayer $\mathrm{WSe}_{2}$ [58]. The method will work particularly well when the excitonic density of states, multiplied by the Boltzmann factor, is strongly peaked at the energy of the indirect $\bar{q}$ exciton. The full $q$ integration can then be replaced by just summing over excitons of wave vector $\bar{q}$. We hope that our work will stimulate further investigations directed toward a full manybody perturbation theory of exciton-phonon coupling.

F.P. and L.W. acknowledge support from the FNR, Luxembourg (Projects No. EXCPHON/11280304 and No. INTER/ANR/13/20/NANOTMD, respectively). A. M.-S. acknowledges the Juan de la Cierva (Grant No. IJCI-2015-25799) program (MINECO, Spain). H. P. C. M. acknowledges the F. R. S.-FNRS through the PDR Grants HTBaSE (T.1071.15). We acknowledge F. Giustino for suggesting the use of the RS relation and $\mathrm{M}$. Zacharias, T. Galvani, M. Barborini, and S. Reichardt for stimulating discussions. We are also indebted to T.Q.P. Vuong, G. Cassabois, B. Gil, and J. Barjon for critical observations about the preliminary results.

Note added in the proof.-Recently, we became aware of a paper, Ref. [59], which is also about phonon-assisted luminescence in $\mathrm{hBN}$ via a finite-difference approach, but with a different description of excited-state occupations.

*Corresponding author. fulvio.paleari@uni.lu

[1] K. Watanabe, T. Taniguchi, and H. Kanda, Nat. Mater. 3, 404 (2004).

[2] Y. Kubota, K. Watanabe, O. Tsuda, and T. Taniguchi, Science 317, 932 (2007).

[3] G. Watanabe, T. Taniguchi, T. Niiyama, K. Miya, and M. Taniguchi, Nat. Photonics 3, 591 (2009).

[4] B. Arnaud, S. Lebègue, P. Rabiller, and M. Alouani, Phys. Rev. Lett. 96, 026402 (2006).

[5] W. Aggoune, C. Cocchi, D. Nabok, K. Rezouali, M. A. Belkhir, and C. Draxl, Phys. Rev. B 97, 241114(R) (2018).
[6] J. S. Lauret, R. Arenal, F. Ducastelle, A. Loiseau, M. Cau, B. Attal-Tretout, E. Rosencher, and L. Goux-Capes, Phys. Rev. Lett. 94, 037405 (2005).

[7] P. Jaffrennou, J. Barjon, J.-S. Lauret, A. Loiseau, F. Ducastelle, and B. Attal-Tretout, J. Appl. Phys. 102, 116102 (2007).

[8] K. Watanabe and T. Taniguchi, Phys. Rev. B 79, 193104 (2009).

[9] C. Attaccalite, M. Bockstedte, A. Marini, A. Rubio, and L. Wirtz, Phys. Rev. B 83, 144115 (2011).

[10] G. Cassabois, P. Valvin, and B. Gil, Nat. Photonics 10, 262 (2016).

[11] G. Cassabois, P. Valvin, and B. Gil, Phys. Rev. B 93, 035207 (2016).

[12] T. Q. P. Vuong, G. Cassabois, P. Valvin, S. Liu, J. H. Edgar, and B. Gil, Phys. Rev. B 95, 201202(R) (2017).

[13] L. J. Martínez, T. Pelini, V. Waselowski, J. R. Maze, B. Gil, G. Cassabois, and V. Jacques, Phys. Rev. B 94, 121405(R) (2016).

[14] L. Schué, L. Sponza, A. Plaud, H. Bensalah, K. Watanabe, T. Taniguchi, F. Ducastelle, A. Loiseau, and J. Barjon, Phys. Rev. Lett. 122, 067401 (2019).

[15] P. Vuong, S. Liu, A. Van Der Lee, R. Cusco, L. Artus, T. Michel, P. Valvin, J. Edgar, G. Cassabois, and B. Gil, Nat. Mater. 17, 152 (2018).

[16] J. Noffsinger, E. Kioupakis, C. G. Van de Walle, S. G. Louie, and M. L. Cohen, Phys. Rev. Lett. 108, 167402 (2012).

[17] C. E. Patrick and F. Giustino, J. Phys. Condens. Matter 26, 365503 (2014).

[18] M. Zacharias, C. E. Patrick, and F. Giustino, Phys. Rev. Lett. 115, 177401 (2015).

[19] M. Zacharias and F. Giustino, Phys. Rev. B 94, 075125 (2016).

[20] Y. Toyozawa, Prog. Theor. Phys. 20, 53 (1958).

[21] G. Antonius and S. G. Louie, arXiv:1705.04245.

[22] B. Segall and G. D. Mahan, Phys. Rev. 171, 935 (1968).

[23] S. Rudin, T. L. Reinecke, and B. Segall, Phys. Rev. B 42, 11218 (1990).

[24] A. Marini, Phys. Rev. Lett. 101, 106405 (2008).

[25] V. Perebeinos, J. Tersoff, and P. Avouris, Phys. Rev. Lett. 94, 027402 (2005).

[26] D. Christiansen, M. Selig, G. Berghäuser, R. Schmidt, I. Niehues, R. Schneider, A. Arora, S. M. de Vasconcellos, R. Bratschitsch, E. Malic, and A. Knorr, Phys. Rev. Lett. 119, 187402 (2017).

[27] A. Chernikov, V. Bornwasser, M. Koch, S. Chatterjee, C. N. Böttge, T. Feldtmann, M. Kira, S. W. Koch, T. Wassner, S. Lautenschläger, B. K. Meyer, and M. Eickhoff, Phys. Rev. B 85, 035201 (2012).

[28] V. Solozhenko, G. Will, and F. Elf, Solid State Commun. 96, 1 (1995).

[29] M. S. Hybertsen and S. G. Louie, Phys. Rev. B 34, 5390 (1986).

[30] F. Paleari, T. Galvani, H. Amara, F. Ducastelle, A. MolinaSánchez, and L. Wirtz, 2D Mater. 5, 045017 (2018).

[31] H. Henck, D. Pierucci, G. Fugallo, J. Avila, G. Cassabois, Y. J. Dappe, M. G. Silly, C. Chen, B. Gil, M. Gatti, F. Sottile, F. Sirotti, M. C. Asensio, and A. Ouerghi, Phys. Rev. B 95, 085410 (2017). 
[32] See Supplemental Material at http://link.aps.org/ supplemental/10.1103/PhysRevLett.122.187401 for computational details, symmetry analysis of excitons and phonons, theoretical support for our formulas, and for additional numerical analysis of the spectra, which includes Refs. [33-43].

[33] P. Giannozzi et al., J. Phys. Condens. Matter 21, 395502 (2009).

[34] J. P. Perdew and A. Zunger, Phys. Rev. B 23, 5048 (1981).

[35] M. Fuchs and M. Scheffler, Comput. Phys. Commun. 119, 67 (1999).

[36] S. Baroni, S. de Gironcoli, A. Dal Corso, and P. Giannozzi, Rev. Mod. Phys. 73, 515 (2001).

[37] D. Sangalli, A. Ferretti, H. Miranda, C. Attaccalite, I. Marri, E. Cannuccia, P. M. Melo, M. Marsili, F. Paleari, A. Marrazzo, G. Prandini, P. Bonfà, M. O. Atambo, F. Affinito, M. Palummo, A. M. Sanchez, C. Hogan, M. Grüning, D. Varsano, and A. Marini, J. Phys. Condens. Matter, https:// doi.org/10.1088/1361-648X/ab15d0 (2019).

[38] V. Hernandez, J. E. Roman, and V. Vidal, ACM Trans. Math. Softw. 31, 351 (2005).

[39] G. Onida, L. Reining, and A. Rubio, Rev. Mod. Phys. 74, 601 (2002).

[40] L. H. Hall, J. Bardeen, and F. J. Blatt, Phys. Rev. 95, 559 (1954).

[41] M. S. Dresselhaus, G. Dresselhaus, and A. Jorio, Group Theory: Application to the Physics of Condensed Matter (Springer, New York, 2008).

[42] F. Bassani and G. P. Parravicini, Electronic States and Optical Transition in Solids (Pergamon, Oxford, 1975).

[43] H. B. Bebb and E. W. Williams, Semiconductors and Semimetals (Elsevier, New York, 1972).

[44] M. Rohlfing and S. G. Louie, Phys. Rev. B 62, 4927 (2000).

[45] A. Davydov, Theory of Molecular Excitons (McGraw-Hill, New York, 1969).

[46] P. Dawson, J. Phys. Chem. Solids 36, 1401 (1975).
[47] J. H. Lloyd-Williams and B. Monserrat, Phys. Rev. B 92, 184301 (2015).

[48] L. Sponza, H. Amara, C. Attaccalite, S. Latil, T. Galvani, F. Paleari, L. Wirtz, and F. Ducastelle, Phys. Rev. B 98, 125206 (2018).

[49] T. Q. P. Vuong, G. Cassabois, P. Valvin, V. Jacques, A. V. D. Lee, A. Zobelli, K. Watanabe, T. Taniguchi, and B. Gil, 2D Mater. 4, 011004 (2017).

[50] $M_{\lambda \bar{q}}=\sum_{\kappa=\mathrm{N}, \mathrm{B}} m_{\kappa}\left|e_{\kappa, \lambda \bar{q}}\right|^{2}$ is the generalized unit cell mass obtained from the normalization condition of phonon eigenvectors $e_{\lambda \bar{q}}$ and atomic masses $m_{\mathrm{B}}$ for boron and $m_{\mathrm{N}}$ for nitrogen.

[51] M. F. Pereira and K. Henneberger, Phys. Rev. B 58, 2064 (1998).

[52] K. Hannewald, S. Glutsch, and F. Bechstedt, Phys. Rev. B 62, 4519 (2000).

[53] P. M. M.C. de Melo and A. Marini, Phys. Rev. B 93, 155102 (2016).

[54] P. T. Landsberg, Recombination in Semiconductors (Cambridge University Press, Cambridge, England, 1991).

[55] W. van Roosbroeck and W. Shockley, Phys. Rev. 94, 1558 (1954).

[56] Our error in $q$ space is approximately $12 \%$ of $\Gamma \mathrm{K}$, with $\Delta q=0.01 \AA^{-1}$. The discrepancy in the position of the LO peak is consistent with the true minimum of the excitonic dispersion lying on the $K$ side of $\bar{q}$. This seems to be confirmed by the computational results in Ref. [14].

[57] T. Q. P. Vuong, G. Cassabois, P. Valvin, V. Jacques, R. Cuscó, L. Artús, and B. Gil, Phys. Rev. B 95, 045207 (2017).

[58] W.-T. Hsu, L.-S. Lu, D. Wang, J.-K. Huang, M.-Y. Li, T.-R. Chang, Y.-C. Chou, Z.-Y. Juang, H.-T. Jeng, L.-J. Li, and W.-H. Chang, Nat. Commun. 8, 929 (2017).

[59] E. Cannuccia, B. Monserrat, and C. Attaccalite, Phys. Rev. B 99, 081109 (2019). 